\title{
Unveiling the enigma of ATLAS17aeu ${ }^{\star, \star \star}$
}

A. Melandri ${ }^{1}$, A. Rossi ${ }^{2}$, S. Benetti ${ }^{3}$, V. D'Elia ${ }^{4,5}$, S. Piranomonte ${ }^{5}$, E. Palazzi ${ }^{2}$, A. J. Levan ${ }^{6}$, M. Branchesi ${ }^{7,8}$, A. J. Castro-Tirado ${ }^{9}$, P. D’Avanzo ${ }^{1}$, Y.-D. Hu ${ }^{9}$, G. Raimondo ${ }^{10}$, N. R. Tanvir ${ }^{11}$, L. Tomasella ${ }^{3}$, L. Amati ${ }^{2}$, S. Campana ${ }^{1}$, R. Carini ${ }^{3}$, S. Covino ${ }^{1}$, F. Cusano ${ }^{2}$, M. Dadina ${ }^{2}$, M. Della Valle ${ }^{12,9}$, X. Fan ${ }^{13}$, P. Garnavich $^{14}$, A. Grado ${ }^{12}$, G. Greco ${ }^{15}$, J. Hjorth ${ }^{16}$, J. D. Lyman ${ }^{6}$, N. Masetti ${ }^{2,17}$, P. O’Brien ${ }^{11}$, E. Pian ${ }^{2}$, A. Perego ${ }^{18,19}$, R. Salvaterra ${ }^{20}$, L. Stella ${ }^{5}$, G. Stratta ${ }^{15}$, S. Yang ${ }^{3}$, A. di Paola ${ }^{5}$, M. D. Caballero-García ${ }^{21}$, A. S. Fruchter ${ }^{22}$, A. Giunta ${ }^{5}$, F. Longo ${ }^{23}$, M. Pinamonti ${ }^{24}$, V. V. Sokolov ${ }^{25}$, V. Testa ${ }^{5}$, A. F. Valeev ${ }^{25}$, and E. Brocato ${ }^{5}$ on behalf of the Gravitational Wave InAf TeAm (GRAWITA)

1 INAF - Osservatorio Astronomico di Brera, Via E. Bianchi 46, 23807 Merate, LC, Italy e-mail: andrea.melandri@brera.inaf.it

2 INAF - Osservatorio di Astrofisica e Scienza dello Spazio di Bologna, Via Piero Gobetti 93/3, 40129 Bologna, Italy

3 INAF - Osservatorio Astronomico di Padova, Vicolo dell'Osservatorio 5, 35122 Padova, Italy

ASI - Science Data Centre, Via del Politecnico snc, 00133 Roma, Italy

INAF - Osservatorio Astronomico di Roma, Via di Frascati, 33, 00040 Monteporzio Catone, Italy

Department of Physics, University of Warwick, Coventry CV4 7AL, UK

7 Gran Sasso Science Institute, Viale F. Crispi 7, 67100 L'Aquila, Italy

INFN - Laboratori Nazionali del Gran Sasso, 67100 L'Aquila, Italy

Instituto de Astrofísica de Andalucía (IAA-CSIC), Glorieta de la Astronomía, s/n, 18008 Granada, Spain

${ }^{10}$ INAF - Osservatorio Astronomico d'Abruzzo, Via Mentore Maggini, 64100 Teramo, Italy

11 Department of Physics and Astronomy, University of Leicester, Leicester LE1 7RH, UK

12 INAF - Osservatorio Astronomico di Capodimonte, Salita Moiariello 16, 80131 Napoli, Italy

13 Steward Observatory, The University of Arizona, 933 North Cherry Avenue, Tucson, AZ 85721-0065, USA

14 Department of Physics, University of Notre Dame, Notre Dame, IN 46556, USA

15 Urbino University, Via Santa Chiara 27, 61027 Urbino, Italy

16 Dark Cosmology Centre, Niels Bohr Institute, University of Copenhagen, Juliane Maries Vej 30, 2100 Copenhagen, Denmark

17 Departamento de Ciencias Físicas, Universidad Andrés Bello, Fernández Concha 700, Las Condes, Santiago, Chile

18 INFN - Sezione Milano Bicocca, Gruppo Collegato di Parma, Parco Area delle Scienze 7/A, 43124 Parma, Italy

19 Dipartimento di Fisica, Universitá degli Studi di Milano Bicocca, Piazza della Scienza 3, 20126 Milano, Italy

${ }^{20}$ INAF - IASF Milano, Via E. Bassini 15, 20133 Milano, Italy

21 Astronomical Institute, Academy of Sciences of the Czech Republic, Bocni II 1401, 14100 Prague, Czech Republic

22 Space Telescope Science Institute, 3700 San Martin Drive, Baltimore, MD 21218, USA

23 Department of Physics, University of Trieste, and INFN, Sezione di Trieste, Via Valerio 2, 34127 Trieste, Italy

24 INAF - Osservatorio Astronomico di Torino, Strada Osservatorio 20, 10025 Pino Torinese, Italy

25 Special Astrophysical Observatory, Nizhnij Arkhyz, Karachai-Cherkessian Republic 369167, Russia

Received 10 July 2018 / Accepted 9 November 2018

\section{ABSTRACT}

Aims. The unusual transient ATLAS17aeu was serendipitously detected within the sky localisation of the gravitational wave trigger GW 170104. The importance of a possible association with gravitational waves coming from a binary black hole merger led to an extensive follow-up campaign, with the aim of assessing a possible connection with GW 170104.

Methods. With several telescopes, we carried out both photometric and spectroscopic observations of ATLAS17aeu, for several epochs, between $\sim 3$ and $\sim 230$ days after the first detection.

Results. We studied in detail the temporal and spectroscopic properties of ATLAS17aeu and its host galaxy. Although at low significance and not conclusive, we found similarities to the spectral features of a broad-line supernova superposed onto an otherwise typical long-GRB afterglow. Based on analysis of the optical light curve, spectrum, and host galaxy spectral energy distribution, we conclude that the redshift of the source is probably $z \simeq 0.5 \pm 0.2$.

Conclusions. While the redshift range we have determined is marginally compatible with that of the gravitational wave event, the presence of a supernova component and the consistency of this transient with the $E_{\mathrm{p}}-E_{\text {iso }}$ correlation support the conclusion that ATLAS17aeu was associated with the long gamma-ray burst GRB 170105A. This rules out the association of the GRB 170105A/ATLAS17aeu transient with the gravitational wave event GW 170104, which was due to a binary black hole merger.

Key words. gamma-ray burst: individual: GRB 170105A - supernovae: general - gravitational waves

\footnotetext{
* Based on observations made with the following telescopes: Copernico, TNG (under programme A34TAC_24), GTC (under programmes GTCMULTIPLE2D-16B and GTCMULTIPLE2G-17A), LBT (under programme 2016_2017_19), and HST (under programme GO14270). $\star \star$ Spectral data for this source shown on this paper are available on the Weizmann Interactive Supernova Data Repository (WISeREP, https://wiserep.weizmann.ac.il/).
} 


\section{Introduction}

The first direct observation of gravitational waves by the Advanced LIGO interferometers came from the coalescence of a binary system of black holes (Abbott et al. 2016a), opening the era of gravitational-wave astronomy. Gravitational-wave signals from binary systems of black holes were detected several times during the first and second run of observations of the advanced detectors (Abbott et al. 2017a,b,c, 2016b). They provided us with information about their rate and mass distribution, and probed their formation and evolution. The first gravitational-wave signal from the inspiral of a binary neutron star system GW 170817 (Abbott et al. 2017d) was observed on August 17, 2017, by the Advanced LIGO and Virgo network, starting the era of multimessenger astronomy (Abbott et al. 2017e).

On January 4, 2017, at 10:11:58.6 UTC the Advanced LIGO detectors revealed the signal from a binary black-hole coalescence, GW 170104 (Abbott et al. 2017a). The system was made of component black holes of masses $31.2_{-6.0}^{+8.4} M_{\odot}$ and $19.4_{-5.9}^{+5.3} M_{\odot}$ (at the $90 \%$ confidence level) at a luminosity distance of $880_{-390}^{+450} \mathrm{Mpc}$ corresponding to a redshift of $z=0.18_{-0.07}^{+0.08}$ (Abbott et al. 2017a). An alert with an initial source localisation $\left(\sim 1600 \mathrm{deg}^{2}\right.$ at the $90 \%$ confidence level) was distributed to collaborating astronomers (The LIGO Scientific Collaboration \& Virgo Collaboration 2017). During the electromagnetic counterpart follow-up search, the ATLAS and Pan-STARRS surveys discovered ATLAS17aeu (Tonry 2017) $23.1 \mathrm{~h}$ after GW 170104, which was a rapidly fading transient within the inner $16 \%$ sky-localisation probability contour (see Fig. 1). The transient, with a decay similar to a GRB afterglow, was also detected in X-rays by the Neil Gehrels Swift Observatory and in the radio at 6 and $15 \mathrm{GHz}$ by the VLA and the AMI large array, respectively (Evans et al. 2017a,b; Corsi 2017; Mooley et al. 2017).

By fitting a power law to the optical decay, the time zero was found consistent with the gamma-ray burst GRB 170105A (Kasliwal et al. 2017) detected by the POLAR instrument on board the Chinese space laboratory Tiangong-2 (Marcinkovski et al. 2017), AstroSat-CZTI (Sharma et al. 2017), KonusWind, and INTEGRAL-SPIACS (Svinkin 2017) $20.04 \mathrm{~h}$ after GW 170104. Temporal and spatial consistency led to the conclusion that ATLAS17aeu was the afterglow of GRB 170105A and unrelated to GW 170104 (Bhalerao et al. 2017a). Considering all the multi-wavelength observations of ATLAS17aeu, Stalder et al. (2017) concluded that the GRB 170105A is compatible with a classical long-GRB at redshift $1 \lesssim z \lesssim 2.9$ and that ATLAS17aeu is statistically likely the associated afterglow. However, they evaluated a small but non-negligible probability of association of ATLAS17aeu and the GW signal, which only a direct redshift measurement of the host galaxy of ATLAS17aeu could exclude.

We present optical observations of ATLAS17aeu transient and its possible host galaxy taken with the $1.8 \mathrm{~m}$ Asiago Copernico telescope, the $3.6 \mathrm{~m}$ Telescopio Nazionale Galileo (TNG), the $8.4 \mathrm{~m}$ Large Binocular telescope (LBT), the $10.4 \mathrm{~m}$ Gran Telescopio Canarias (GTC), and the Hubble Space Telescope (HST) over 230 days from the GRB 170105A trigger time. Together with the radio and X-ray observations, the connection between the transient ATLAS17aeu and the long gamma-ray burst GRB 170105A is discussed.

Throughout the paper, distances are computed assuming a $\Lambda$ CDM-Universe with $H_{0}=71 \mathrm{~km} \mathrm{~s}^{-1} \mathrm{Mpc}^{-1}, \Omega_{\mathrm{m}}=0.27$, and $\Omega_{\Lambda}=0.73$ (Larson et al. 2011; Komatsu et al. 2011).

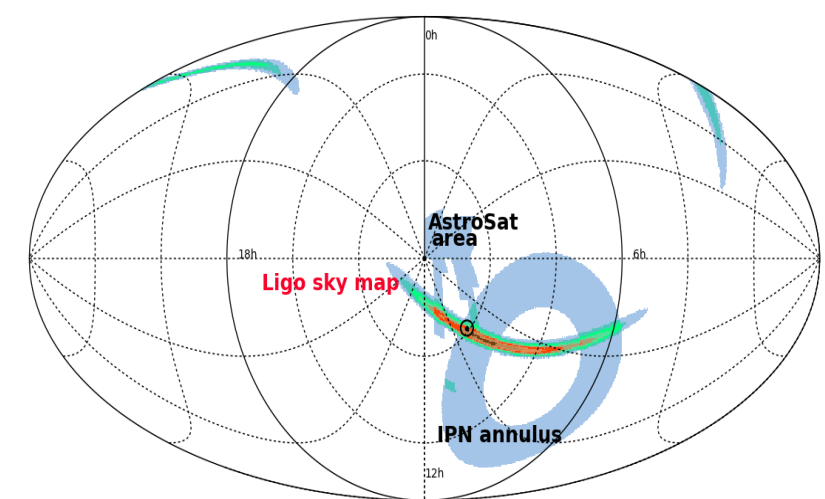

Fig. 1. Mollweide projection for localisation area of ATLAS17aeu (black circle). We report the AstroSat CZTI localisation area at a $1 \sigma$ confidence level and the IPN triangulation annulus at a $3 \sigma$ confidence level for GRB 170105A (light blue). The final LVC sky map for GW 170104 is also shown.

Magnitudes are in the $\mathrm{AB}$ system and errors are at a $1 \sigma$ confidence level.

\section{ATLAS17aeu}

The rapidly fading transient ATLAS17aeu (Tonry 2017), identified within the localisation of GW 170104 (The LIGO Scientific Collaboration \& Virgo Collaboration 2017), was only $20^{\prime \prime}$ away from the SDSS galaxy J091312.36+610554.2, with a spectroscopic redshift $(z \sim 0.2)$ consistent with the distance inferred for GW 170104. Considering this galaxy as the possible host of ATLAS17aeu, the position and distance consistency of ATLAS17aeu and GW 70104 led to many multiwavelength observations to probe the possible association of ATLAS17aeu with the gravitational signal.

Within the GRAWITA ${ }^{1}$ framework, we monitored the light curve of the source between 1.65 and 88.7 days after the GRB trigger with the Asiago and TNG telescopes. In addition, we obtained two multi-filter epochs ( $\mathrm{griz}$ ) with the GTC telescope between 3.92 and 78.7 days after the GRB trigger. Finally, we observed the field with the LBT telescope in imaging mode (gri) at $\sim 104$ days after the gamma-ray burst trigger, GRB 170105A. Image reduction was carried out following standard procedures and the optical data were calibrated using a common set of selected catalogued stars of the SDSS catalogue present in the field of view.

Two spectra were also acquired with the GTC telescope, one at $\sim 3$ days and a second secured at $\sim 116$ days after the burst event. GTC spectroscopy was carried out using the OSIRIS camera in slit mode, with the $\mathrm{R} 1000 \mathrm{~B}(R=1000$, spectral range 3630-7500 $\AA$ ) and $\mathrm{R} 2500 \mathrm{I}(R=2500$, spectral range 7330-10000 ̊) grisms. The slit width was set to $1^{\prime \prime}$. The data were optimally extracted (Horne 1986) and reduced following standard procedures using ESO MIDAS ${ }^{2}$ and IRAF $^{3}$ software.

In addition, three further optical spectra were collected on April 14, 2017, on January 25, 2018, and on March 19, 2018, with LBT using the two Multi-Object Double Spectrograph (MODS, Pogge et al. 2010). All observations were obtained in the spectral range $3200-9500 \AA$ with a $1^{\prime \prime}$ slit $(R \sim 2000)$.

\footnotetext{
1 GRAvitational Wave Inaf TeAm: https://www.grawita.inaf. it/

2 http://www.eso.org/projects/esomidas/

3 http://iraf.noao.edu/
} 
MODS uses two red- and blue-optimised channels with a spectral range of 3500-6500 $\AA$ and 5000-10000 The first two epochs were taken with the one grating for each channel; this has the advantage of avoiding a gap at $\sim 5650 \AA$ between the two channels, but doubles the observing time. Therefore, the last epochs were taken with the dual grating mode in which the light is separated by a dichroic into red- and bluechannels. A $2 \times 2$ binning was set in the second epoch, but it caused read-out artefacts and thus in the final epoch we adopted a $1 \times 2$ binning. The last observation was obtained under the best conditions (seeing $\sim 0.7^{\prime \prime}$, airmass $1.1-1.2$ ) for a total exposure time of $4800 \mathrm{~s}$. Data reduction was performed at the Italian LBT Spectroscopic Reduction Center ${ }^{4}$ by means of scripts optimised for LBT data. The data reduction steps of each two-dimensional spectral image are the correction for dark and bias, bad-pixel mapping, flat-fielding, sky background subtraction, and extraction of the one-dimensional spectrum by integrating the stellar trace along the spatial direction. Wavelength calibration was obtained from the spectra of arc lamps, while calibration was obtained using catalogued spectrophotometric standards.

The location of ATLAS17aeu was subsequently observed with the HST-WFC on August 22, 2017 ( 229 days after the burst event). At this point, observations were obtained in the UVIS arm F390W, F606W and the IR arm with F140W 5 . Observations were reduced by astrodrizzle in the standard fashion. At the location of ATLAS17aeu, we clearly detect a source in both F606W and F140W, but there is no detection in F390W (Fig. 3). The position of the ATLAS17aeu transient is RA $=09: 13: 13.89$, Dec $=+61: 05: 32.54$ with an error of $0.06^{\prime \prime}$.

For our UVIS observations, we measure the AB magnitudes (or upper limits) within a $0.1^{\prime \prime}$ aperture and correct them with the published encircled energy curves ${ }^{6}$. We determine that F390W > $28.1 \mathrm{mag}(3 \sigma)$ and F606W $=27.64 \pm 0.21 \mathrm{mag}$. In the IR we use a $0.2^{\prime \prime}$ aperture due to the poorer PSF, and measure $\mathrm{F} 140 \mathrm{~W}=25.87 \pm 0.14 \mathrm{mag}$. There is no sign of extension in the images, and the sources appear point-like. However, at this faint magnitude the detection of extension is challenging. We consider the source located S-E with respect to ATLAS17aeu as its host galaxy. The separation between the two objects is $\sim 1.8^{\prime \prime}$ (Fig. 3).

The summary of our photometric and spectroscopic observations is given in Tables 1 and 2, respectively. Data have not been corrected for Galactic extinction $\left(E_{B-V}=0.028 \mathrm{mag}\right.$, Schlafly \& Finkbeiner 2011).

\section{GRB 170105A}

GRB 170105A was detected at 06:14:07.0 UT ( $T_{0}$, corresponding to MJD 57758.259803) with a total duration $T_{90}=2.0 \pm 0.5 \mathrm{~s}$. The burst event was also detected by INTEGRAL-SPIACS, Konus-Wind, and AstroSat-CZTI (Sharma et al. 2017) with a measured duration $T_{90} \sim 2.9 \mathrm{~s}$. Its fluence derived from the Konus-Wind observation is $S_{[0.02-10 \mathrm{MeV}]} \sim 2.5 \times 10^{-6} \mathrm{erg} \mathrm{cm}^{-2}$ and it displayed longer emission (with a duration of about $20 \mathrm{~s}$ ) in the $18-70 \mathrm{keV}$ soft channel of Konus-Wind (Svinkin 2017; Stalder et al. 2017).

In Fig. 1 we show the localisation areas (AstroSat and IPN) for this event, together with the LVC sky map for GW 170104 and the most accurate position for ATLAS17aeu. As it can be seen ATLAS17aeu is slightly outside the $1 \sigma$ AstroSat/CZTI

\footnotetext{
4 http://www.iasf-milano.inaf.it/Research/lbt_rg.html

5 HST observations have been cross-calibrated with the $g, r$, and $J$ bands, respectively.

6 http://www.stsci .edu/hst/wfc3/phot_zp_lbn
}

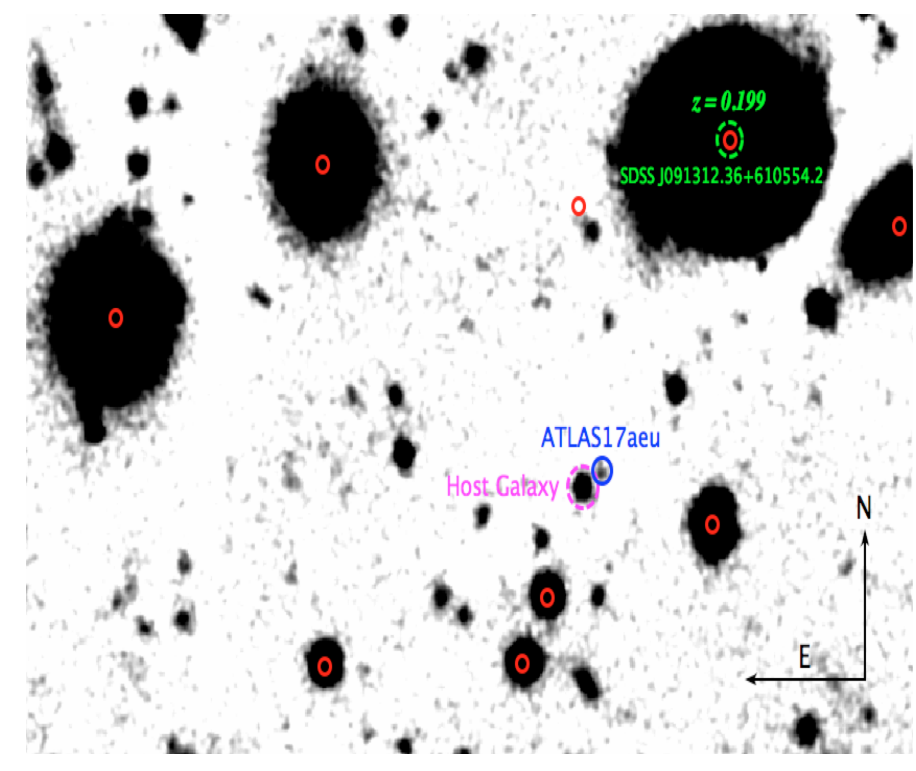

Fig. 2. TNG image (field of view $\sim 1^{\prime} \times 1^{\prime}$ ) acquired at $\Delta t \sim 56$ days, in the $r$ filter. The ATLAS17aeu position (blue circle) is $\sim 2^{\prime \prime}$ away from the centre of its host galaxy (magenta circle). Shown are the catalogued SDSS objects (red circles), including the J091312.36+610554.2 galaxy at $z \sim 0.2$ (green circle).

localisation area, well within the LVC probability contours. The temporal and spatial coincidence between ATLAS17aeu and GRB 170105A indicated that the two events were most likely associated (Kasliwal et al. 2017; Bhalerao et al. 2017a,b) while it remained unclear the association with the GW 170104 due to the lack of a firm ATLAS17aeu distance determination.

The GRB 170105A fluence is consistent with a long-soft, under-energetic GRB. In fact, assuming the distance inferred for GW $170104(z \sim 0.1)$ the estimated isotropic energy of the GRB event would be $E_{\text {iso }} \sim 5.8 \times 10^{49} \mathrm{erg}$, and at larger distances up to $z \approx 1$, the isotropic energy remains still consistent with the faint end of the $E_{\text {iso }}$ distribution for long GRBs (Nava et al. 2012; D'Avanzo et al. 2012).

\section{Results and discussion}

\subsection{Temporal analysis}

The early time optical light curve of ATLAS17aeu (Fig. 4) can be described by a single power-law decay $\left(\alpha_{r}=1.38 \pm 0.02\right)$. At a later time ( $>10$ days) a significant deviation from that decay is detected, revealing the presence of a possible supernova component (Fig. 4).

Many known under-energetic long-duration gamma-ray bursts and X-ray flashes have an associated highly strippedenvelope core-collapse supernova (Type Ib/c). At low redshifts $(z \lesssim 0.3)$ the supernova component is clearly identified both photometrically and spectroscopically (Galama et al. 1998; Patat et al. 2001; Hjorth et al. 2003; Malesani et al. 2004; Ferrero et al. 2006; Pian et al. 2006; Cano et al. 2011a; Bufano et al. 2012; Melandri et al. 2012, 2014; Schulze et al. 2014; D'Elia et al. 2015), while at higher redshifts $(0.3 \lesssim z \lesssim 1)$ the presence of the supernova is inferred from the detection of a rebrightening in the late afterglow light curve (Bloom et al. 1999; Castro-Tirado \& Gorosabel 1999; Galama et al. 2000; CastroTirado et al. 2001; Della Valle et al. 2003, 2006; Greiner et al. 2003; Zeh et al. 2004; Soderberg et al. 2006; Cano et al. 2011b; Sparre et al. 2011; Jin et al. 2013). The supernova origin for 

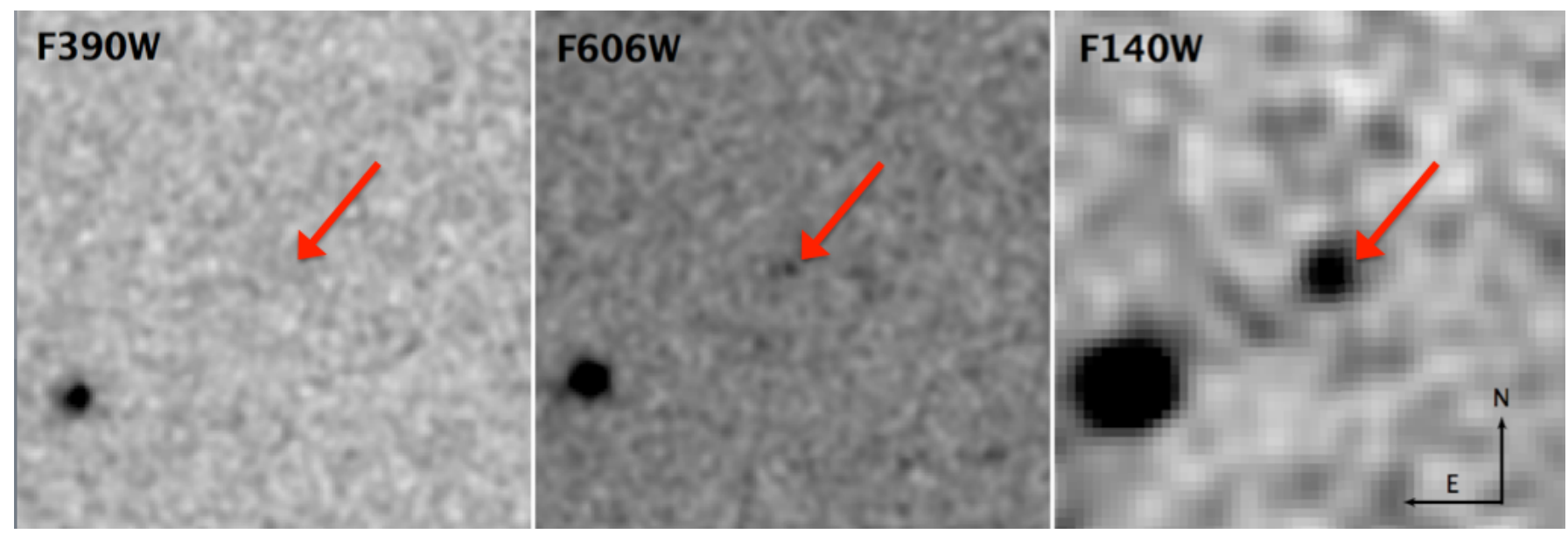

Fig. 3. HST observations of the field of ATLAS17aeu (field of view $=5^{\prime \prime} \times 5^{\prime \prime}$ ). The red arrow indicates the location of the optical transient. The other object visible in that image is what we consider the host galaxy of ATLAS17aeu, reported also in Fig. 2. The offset between the two objects is $\sim 1.8^{\prime \prime}$.

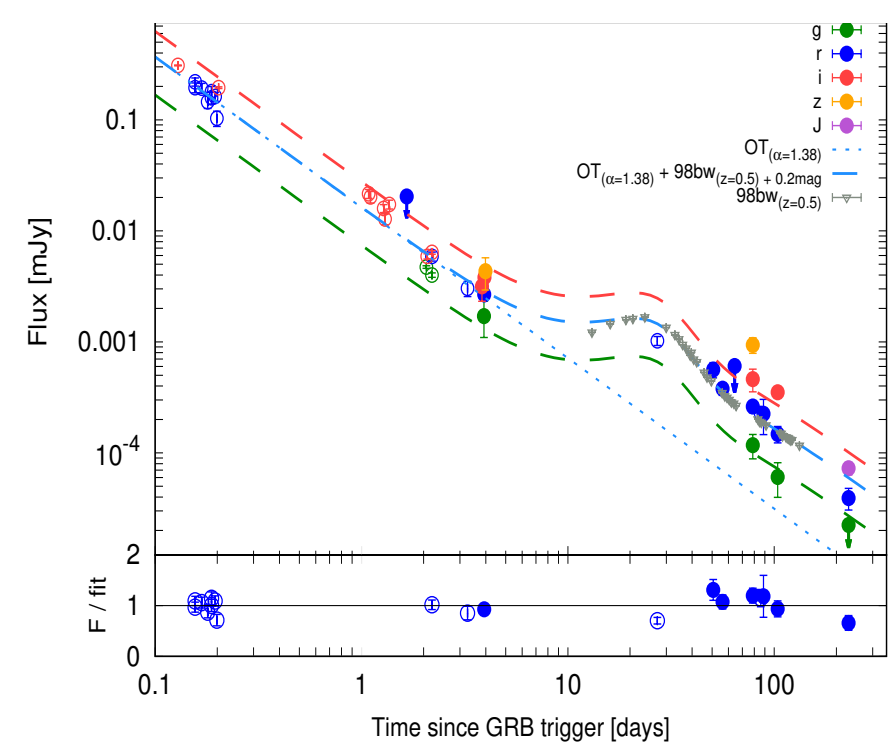

Fig. 4. Optical light curve for ATLAS17aeu. Filled points identify our data, while empty symbols are data from the literature. The power-law decay of the optical afterglow (blue dotted line) and the SN 1998bw (grey open triangles) template at $z=0.5$ are shown. The overall fit to the light curve, assuming SN $1998 \mathrm{bw}$ as a template (fainter by $0.2 \mathrm{mag}$ ) is shown (blue dashed line). The overall fit is then shifted arbitrarily to guide the eye and match the $g$-band (green dashed line) and $i$-band (red dashed line) data.

the re-brightening observed in the afterglows of high- $z$ GRBs is sometimes further enhanced by sporadic spectroscopic observations of the "bumps" that reveal supernova features (e.g. Jin et al. 2013). Our Fig. 4 shows a faint optical light curve of ATLAS17aeu suggesting $z>0.3$ and the signature of an emerging supernova which starts to outshine the GRB afterglow from $\sim 10$ to 12 days.

In order to have additional information about the possible supernova (SN) component we rescaled the absolute $r$-band magnitudes of ATLAS17aeu to the distance of several wellknown Type $\mathrm{Ib} / \mathrm{c} \mathrm{SNe}$, and compared our data with their light curves that cover a wide range of brightness (Fig. 5). This results in a possible range of distances that can explain the observed late time afterglow re-brightening $(0.28 \leq z \leq 0.72)$

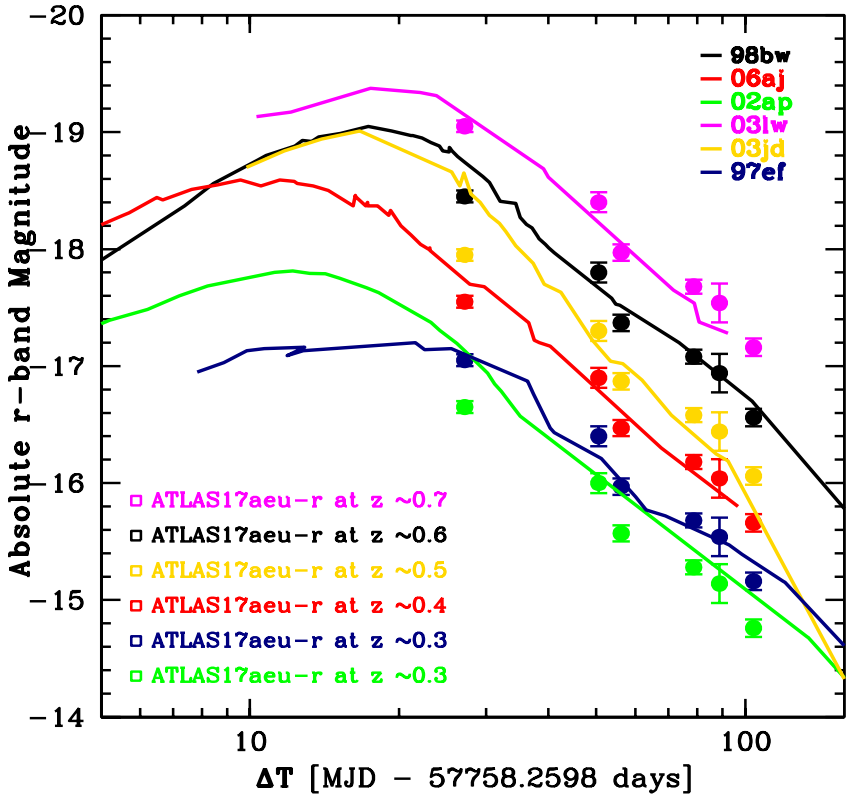

Fig. 5. Comparison between the absolute $r$-band magnitudes of several well-known Type Ib/c SNe and ATLAS17aeu observations. Times are in days since GRB 170105A trigger time and magnitudes have been $k$-corrected. From the match with each $\mathrm{SNe}$ light curve we estimated the possible distance modulus of ATLAS17aeu and infer the possible range of redshifts $(0.3 \leq z \leq 0.7)$ for the transient.

of ATLAS17aeu. The best match is obtained with SN 1998bw (a typical Type $\mathrm{Ib} / \mathrm{c} \mathrm{SN}$ associated with the subluminous gammaray burst GRB 980425) assuming a redshift of $z \sim 0.6$ (see Fig. 4). We note that even in the case of the match with the brightest known SN associated with a GRB (SN 20031w) we obtain $z \sim 0.7$. The hypothesis of a higher redshift would require a much more luminous SN that has never been observed in association with a long GRB.

\subsection{Spectral analysis}

In an attempt to identify a possible SN signature in the GTC spectrum we compared it with several Type Ib/c supernova templates. Its modest quality limits the significance of any match 

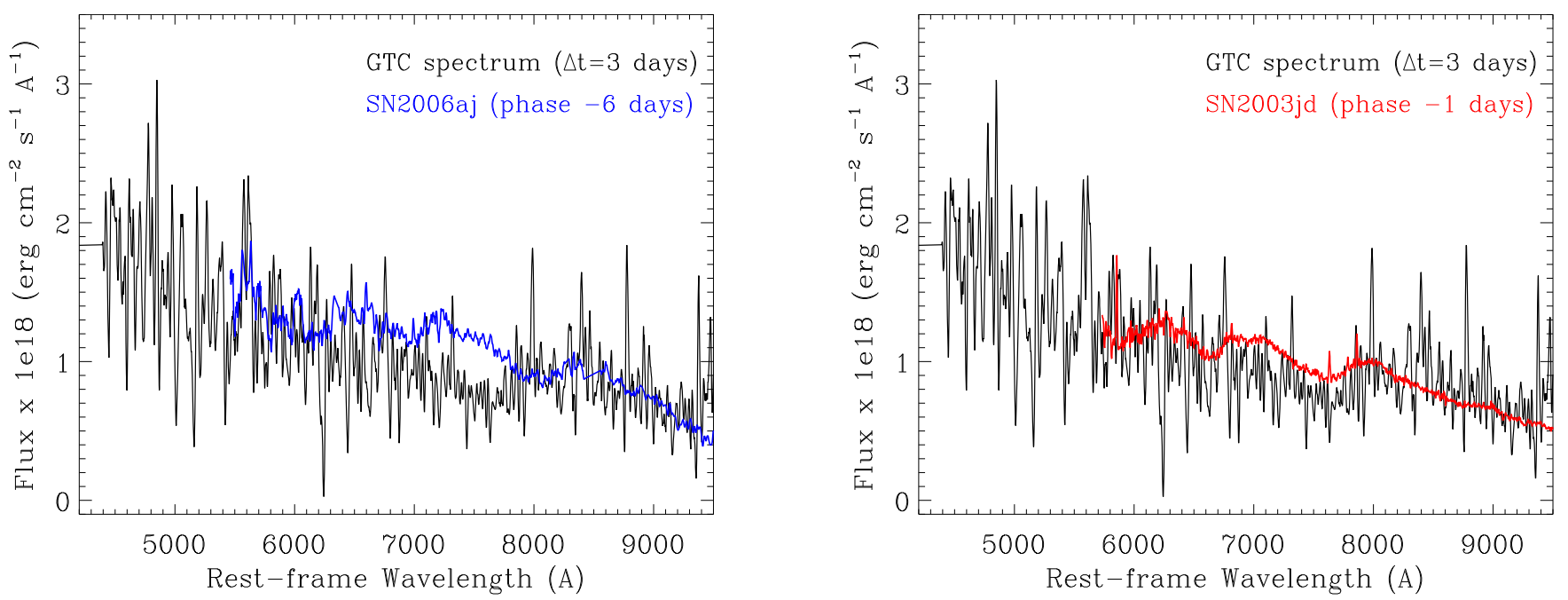

Fig. 6. Left panel: comparison between the early time GTC spectrum of ATLAS17aeu (acquired $\sim 3$ days after the burst event and without removing the afterglow contribution) and the template (assuming a redshift $\sim 0.6 \pm 0.1$ ) of the well-studied Type Ib/c SN 2006aj (Pian et al. 2006) at a phase of $\sim 3$ days after the burst event GRB 060218. Right panel: same assumption as for the left panel, using the template of the Type Ic SN 2003jd (Valenti et al. 2008) at a phase of about one day before B-maximum light.

and, at the time of the observation, the supernova component contributes to less than $10 \%$ to the measured flux (see Fig. 4). Nevertheless, there is some resemblance to broad-line Type Ic SNe 2003jd (Valenti et al. 2008) and 2006aj (e.g. Pian et al. 2006), the latter associated with an under-energetic longduration GRB 060218 (Campana et al. 2006), which points to a redshift of redshift $z \sim 0.6$ (Fig. 6).

A similar value for the redshift is also found when comparing a low S/N LBT spectrum of the very faint host galaxy, taken well after the explosive event, with the template of a starforming galaxy, by identifying possible Balmer transitions at redshift $z \sim 0.6$. The red region of the spectrum (with the signalto-noise ratio of $\sim 4$ ) shows a possible correspondence between the observed lines $(\mathrm{H} \eta, \mathrm{H} \& \mathrm{~K}, \mathrm{H} \delta$, and Gband $+\mathrm{H} \gamma+\mathrm{Fe} 4383 \AA$ ) and the model. These spectral comparisons indicate a plausible redshift for ATLAS17aeu of $z \sim 0.6 \pm 0.1$.

\subsection{Spectral energy distribution of the afterglow}

In order to study the transient, we modelled and investigated the dataset separately at different wavelength ranges: radio, optical, and X-ray. We then interpolate the data to two common epochs and performed a broad-band analysis.

We first fitted the radio light curve, for which there are observations at different frequencies, with most of the data at $15.5 \mathrm{GHz}$ and only a couple of detections at 7.4 and $5.0 \mathrm{GHz}$ (Bhalerao et al. 2017a). The light curve has a different behaviour in the different bands, and decays more rapidly at higher frequencies (Fig. 7, left panel). When we modelled the data with a power-law fit, we obtained decaying indices of $\alpha_{15.5 \mathrm{GHz}}=$ $0.66 \pm 0.04, \alpha_{7.4 \mathrm{GHz}}=0.4$ and $\alpha_{5.0 \mathrm{GHz}}=0.05$ at $15.5,7.4$ and $5.0 \mathrm{GHz}$, respectively. We note that for the latter two indexes there are the same number of data points as parameters and no uncertainty can be provided. The different decays in the radio bands might be due to colour evolution, which can only be explained by the presence of a spectral break moving from higher to lower frequencies.

Then, to study the behaviour in the optical band, we considered the data up to 5 days after the trigger when only the afterglow is contributing to the observed flux. The data were corrected for foreground Galactic extinction. The light curve is best followed up in the $r$ and $i$ filters, with $g$ - and $z$-band data starting only 2 days after the trigger. We studied the griz spectral energy distribution of the afterglow at 3.93 days for which we have detections in all the optical bands. We find that the data are best modelled by a power law with spectral index $\beta_{\text {opt }}=1.21 \pm 0.01$ and negligible dust extinction along the line of sight. Afterwards, we modelled all the optical light curves together and sampled the time and wavelength plane with a twovariable power law $F(v, t) \propto t^{-\alpha_{\mathrm{opt}}} v^{-\beta_{\mathrm{opt}}}$, and fixed $\beta_{\mathrm{opt}}$ to the value reported above. This approach is only possible given the negligible dust extinction. In this way, we find an optical decay common to all optical bands of $\alpha_{\mathrm{opt}}=1.38 \pm 0.02$.

Afterward, we downloaded and reduced the Swift-XRT data. The data span the interval between $\sim 1$ and $\sim 15$ days and can be best modelled by a single power-law model with a decay $\alpha_{\mathrm{X}}=0.87 \pm 0.24$. The X-ray spectrum is rather poor and can be fitted using Xspec v12.9.0 with a simple power law with $\beta=$ $0.7 \pm 0.1$, fixed foreground Galactic absorption $\left(0.66 \times 10^{21} \mathrm{~cm}^{-2}\right.$; Willingale et al. 2013), and negligible host gas absorption.

Finally, we modelled all optical, radio, and X-ray spectral energy distribution (SED) at the logarithmic mean time of the XRT observations, i.e. $~ 3.28$ days. We also selected another epoch at 2.14 days for which we have optical gri detections. We interpolated radio and optical data to the first epoch, and radio and XRT data to the second epoch. In the following we fixed the optical spectral slope to the value $\beta_{\text {opt }}=1.21$ found above. We modelled the SED at 3.28 days with a double broken power law, and we found two spectral breaks: a first spectral break in the radio bands at $(0.7 \pm 0.1) \times 10^{10} \mathrm{~Hz}$ and a second break between radio and optical bands at $(1.0 \pm 0.1) \times 10^{12} \mathrm{~Hz}$. Following the standard synchrotron theory under slow cooling regime (Sari et al. 1998), we identify the first break in the radio as the absorption frequency $v_{a}$, and the break between optical and radio to be the injection frequency $v_{m}$. The slope between $v_{a}$ and $v_{m}$ was fixed to the value of $1 / 3$. It is important to note here that the first break $v_{a}$ is evolving with time and that the decay below the break is almost negligible. This behaviour can be interpreted within the jet scenario (Sari et al. 1999) and the slow cooling regime, which indeed predicts $\alpha=0$ for $v<v_{a}$ and 


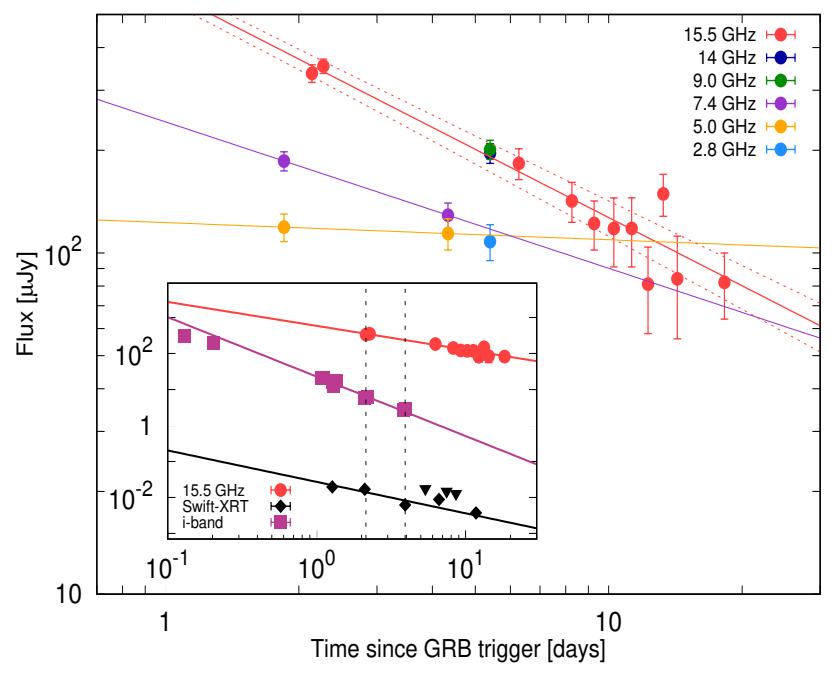

Fig. 7. Radio band light curves of ATLAS17aeu at different frequencies. The data at 5.0 (gold), 7.4 (purple), and 15.5 (red) GHz are fitted with simple power laws. Inset plot: radio (red), optical (plum), and X-ray (black) light curves. Black triangles represent upper limits in the X-ray band. They can all be modelled with simple power laws. Dashed vertical lines represent the selected times for the spectral energy distribution fitting shown in Fig. 8.

$v_{a} \propto t^{-1 / 5}$. Thus, to obtain the model at 2.14 days we followed Sari et al. (1999) and used the relations $v_{a} \propto t^{-1 / 5}$ and $v_{m} \propto t^{-2}$.

In Fig. 8 we show the radio, optical, and X-ray SEDs at different epochs. The fit is acceptable, but it should be noted that the model does not perfectly match the optical data at the first epoch and the jet scenario would predict more rapid decay in optical and X-ray bands. This suggests the presence of a second break between the optical and X-ray, and thus a more sophisticated analysis is needed to fully understand the afterglow behaviour. This can be seen in Fig. 7 (right panel), which shows radio, optical, and X-ray light curves. In particular, while the radio and the X-ray light curves agree within $1 \sigma$ (due to the large uncertainty of $\alpha_{\mathrm{X}}$ ), the optical light curve is not consistent with the others. We interpret this as the presence of another spectral break between optical and X-ray bands. An evolving break between the optical and X-ray can be seen in the jet scenario without sideways expansion if the circumburst medium has a wind profile, and the synchrotron cooling frequency $v_{c}$ lies between the optical and X-ray bands implying $\beta_{\mathrm{X}}-\beta_{\text {opt }}=0.5$, consistent with the values reported above (e.g., Racusin et al. 2009; Schulze et al. 2011). It is also assumed that $\alpha_{\mathrm{opt}}-\alpha_{\mathrm{X}}=0.25$, and indeed by doing so the two decay indexes are consistent within $2 \sigma$.

\subsection{Spectral energy distribution of the host galaxy}

As reported in Sect. 2, the putative faint host-galaxy is located $\sim 1.8^{\prime \prime} \mathrm{S}-\mathrm{E}$ with respect to ATLAS17aeu. Assuming an average redshift $z \sim 0.5$ this separation translates into a project offset of $\sim 11 \mathrm{kpc}$. This distance is slightly larger than typically found for broad-line SNe with and without accompanying long GRB (Japelj et al. 2018).

We used the photometric SED-fitting code LePHARE ${ }^{7}$ (Arnouts et al. 1999; Ilbert et al. 2006) to determine host-galaxy parameters from the detections. After fixing the redshift to the most plausible value found in our spectral and temporal analysis $(z \sim 0.6$, see Sects. 4.1 and 4.2), we found that the host is a

\footnotetext{
7 http://www.cfht.hawaii.edu/ arnouts/LEPHARE
}

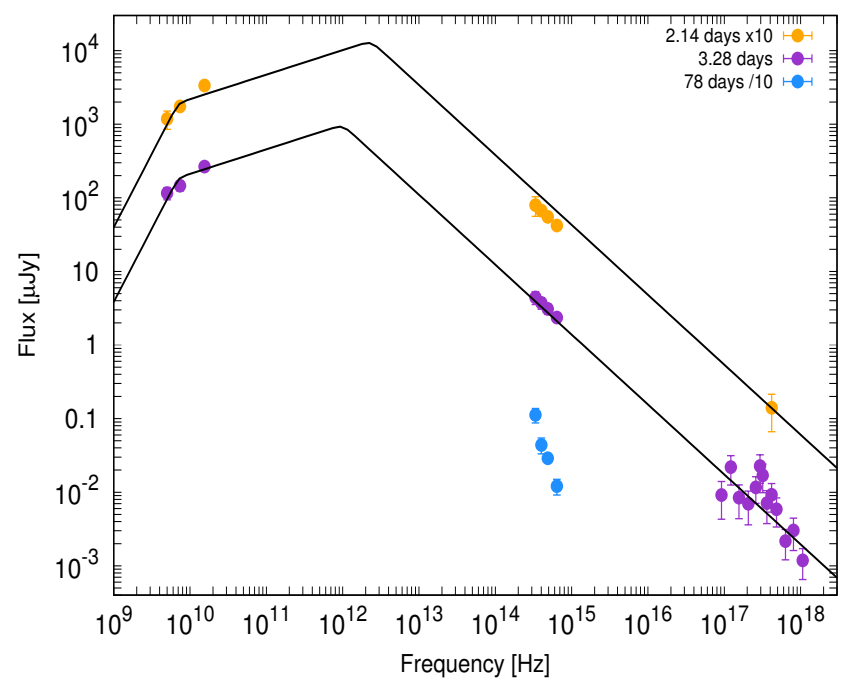

Fig. 8. Radio, optical, and X-ray data at 3.28 (purple) and 2.14 days (gold) of ATLAS17aeu. The model described in the text is also shown at both epochs. Data at 78 days (cyan) are clearly dominated by the supernova emission and we show them only for comparison with previous epochs.

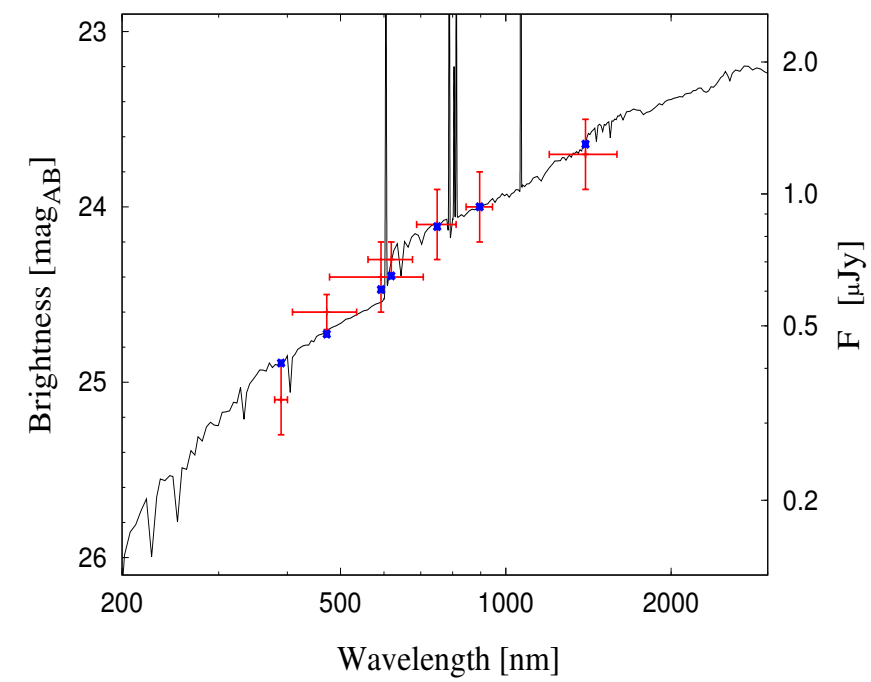

Fig. 9. LePHARE fit to the magnitudes of the host galaxy of ATLAS17aeu/GRB 170105A with the redshift fixed to be the same as the spectroscopic value. The photometric points are highlighted in red, and the blue marks represent the photometry values as determined by the synthetic SED. The fit is acceptable with $\chi / N_{\text {filters }}=2.97 / 7$. For specific values, see text.

low-mass $\left(M=10^{8.2_{-0.2}^{+0.2}} M_{\odot}\right)$ galaxy with low global extinction $\left(E_{B-V} \sim 0.2 \mathrm{mag}\right.$ using Calzetti et al. 2000 extinction law), and low star formation rate $\left(\mathrm{SFR}=0.9_{-0.4}^{+1.5} M_{\odot} \mathrm{yr}^{-1}\right)$. The inferred low mass is in agreement with the mass of typical long GRB hosts at these redshifts (Vergani et al. 2015).

Despite the low SFR, the low mass does not qualify this galaxy as an early type, which would be very unusual for the host of a long GRB (but see Rossi et al. 2014). In fact, the main stellar population is moderately young (age $=0.3_{-0.2}^{+0.6} \mathrm{Gyr}$ ) and the galaxy has a high specific SFR of $10^{-8.3_{-0.4}^{+0.5} \mathrm{yr}^{-1}}$ in agreement with other GRB hosts and star-forming galaxies (Hunt et al. 2014; Japelj et al. 2016). The result of our fit is shown in Fig. 9. It is worth noting that if we do not fix the redshift we can use photometric data to constrain it between $0.4<z<2.8$, which 


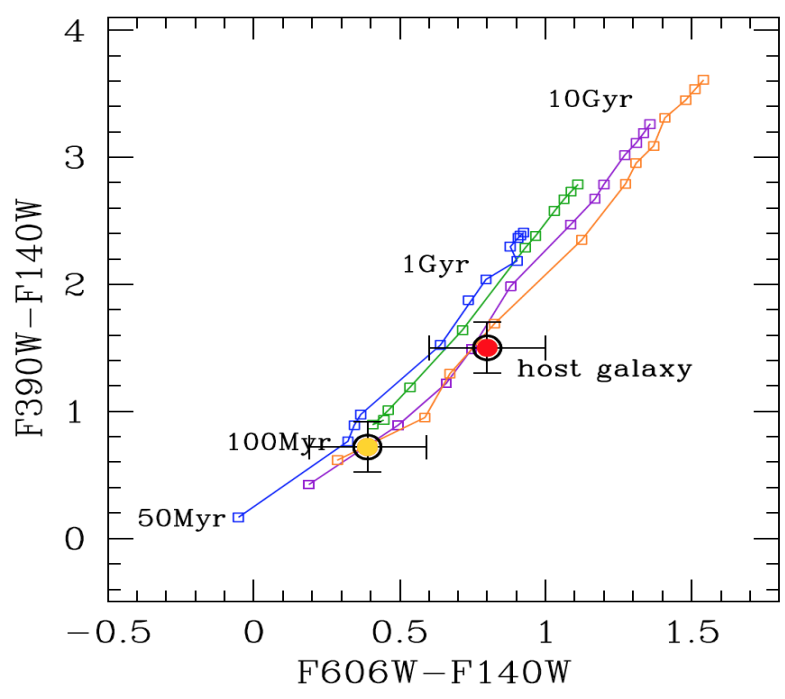

Fig. 10. Distance-independent two-colour diagram. Simple stellar population colours from the SPoT code are compared to the measured hostgalaxy colors (filled yellow circle). Lines and small squares refer to models with metallicity $[\mathrm{Fe} / \mathrm{H}]=-0.7$ (blue), $[\mathrm{Fe} / \mathrm{H}]=-0.4$ (green), $[\mathrm{Fe} / \mathrm{H}]=0.0$ (violet), $[\mathrm{Fe} / \mathrm{H}]=+0.4$ (orange), respectively. Indicative ages are also labelled, from $50 \mathrm{Myr}$ to $14 \mathrm{Gyr}$. The host galaxy is plotted as a filled red (observed) and yellow (de-reddened) circle. The last value is obtained by applying the value $E(B-V)=0.2)$. No $k$-correction is applied.

is expected given the featureless SED and still inconsistent with the inferred distance of GW 170104.

To give some indications of the properties of the host galaxy, in Fig. 10 we also plot the (F390-F140W) colour versus the (F606-F140W) colour of the host galaxy, together with stellar population models. The integrated colour predictions shown in the figure are based on the Stellar Population Tools (SPoT) code for single-age, single-metallicity stellar population (SSP) models (Brocato et al. 1999; Raimondo 2009), updated for this study using higher total stellar masses, and new spectral libraries for cool and hot stars. Models suggest that the main component of the stellar population in the galaxy is as young as a few tens of Myr or younger, in agreement with a high specific SFR (e.g. Feulner et al. 2005). This is mildly in agreement with the results of our photometric host-galaxy SED-fitting. The model and data uncertainties do not permit clear indications of the chemical composition of the stellar content.

\section{Conclusion}

Our optical observations allowed us to comprehensively describe the temporal behaviour of the unusual transient ATLAS17aeu from early to very late phases. The overall spectral behaviour is reminiscent of a broad-line Ic supernova, suggesting that ATLAS17aeu is indeed the optical afterglow of the long-duration under-energetic GRB 170105A, and definitely not associated with the gravitational wave signal GW 170104, which was due to a binary BH merger (Abbott et al. 2017a).

The presence of the supernova is suggested at early times in our first spectrum ( $\sim 3$ days after the burst event) and confirmed at later times by the typical bump in the light curve already seen in many other light curves of GRBs connected SNe. Although the redshift for this event is not strongly constrained by the data, we can confidently define a small range of possible values: $z \simeq 0.5 \pm 0.2$. The temporal behaviour of such a supernova is
Table 1. Imaging log for ATLAS17aeu.

\begin{tabular}{cccccl}
\hline \hline $\begin{array}{c}\text { MJD } \\
(\mathrm{d})\end{array}$ & $\begin{array}{c}\Delta t^{a} \\
(\mathrm{~d})\end{array}$ & $\begin{array}{c}t_{\text {exp }} \\
(\mathrm{min})\end{array}$ & Filt. & Mag (err) & Tel. \\
\hline 57762.179 & 3.92 & 8 & $g$ & $23.53(0.08)$ & GTC \\
57836.964 & 78.7 & 10 & $g$ & $26.34(0.26)$ & GTC \\
57862.194 & 103.9 & 60 & $g$ & $27.05(0.31)$ & LBT \\
57987.330 & 229.1 & 45.6 & F390W & $>28.1$ & HST \\
57820.050 & 61.8 & 80 & $V$ & $>22.6$ & TNG \\
57759.914 & 1.65 & 15 & $r$ & $>20.7$ & Asiago \\
57762.187 & 3.93 & 10 & $r$ & $23.13(0.06)$ & GTC \\
57808.852 & 50.6 & 70 & $r$ & $24.65(0.18)$ & TNG \\
57814.436 & 56.2 & 65 & $r$ & $25.03(0.15)$ & TNG \\
57822.567 & 64.3 & 90 & $r$ & $>25.0$ & TNG \\
57836.973 & 78.7 & 10 & $r$ & $25.43(0.12)$ & GTC \\
57846.960 & 88.7 & 140 & $r$ & $25.48(0.29)$ & TNG \\
57862.194 & 103.9 & 60 & $r$ & $26.05(0.15)$ & LBT \\
57987.330 & 229.1 & 24.4 & F606W & $27.64(0.21)$ & HST \\
57761.093 & 3.84 & 16 & $I$ & $22.52(0.32)$ & TNG \\
57762.197 & 3.94 & 10 & $i$ & $22.85(0.10)$ & GTC \\
57836.953 & 78.7 & 12 & $i$ & $24.98(0.26)$ & GTC \\
57862.178 & 103.9 & 60 & $i$ & $25.13(0.12)$ & LBT \\
57762.205 & 3.94 & 7 & $z$ & $22.67(0.13)$ & GTC \\
57836.980 & 78.7 & 8 & $z$ & $24.01(0.24)$ & GTC \\
57987.330 & 229.1 & 16.8 & F140W & $25.87(0.14)$ & HST \\
\hline
\end{tabular}

Notes. The columns are as follows: modified Julian date (1) and $\Delta t$ corresponding to the mid time of the observation (2), total exposure time (3), filter identification (4), calibrated AB magnitude not corrected for Galactic extinction (5), and telescope used for the observation (6). ${ }^{(a)}$ This time is estimated from $T_{0}$.

Table 2. Spectroscopic log for ATLAS17aeu.

\begin{tabular}{cccccc}
\hline \hline $\begin{array}{c}\text { MJD } \\
(\mathrm{d})\end{array}$ & $\begin{array}{c}\Delta t^{a} \\
(\mathrm{~d})\end{array}$ & $\begin{array}{c}t_{\mathrm{exp}} \\
(\mathrm{min})\end{array}$ & grism & $\begin{array}{c}\text { Seeing } \\
\left({ }^{\prime \prime}\right)\end{array}$ & Tel. \\
\hline 57761.110 & 2.89 & $2 \times 20$ & R1000B & 1.5 & GTC \\
57761.153 & 2.93 & $2 \times 20$ & R2500I & 1.5 & GTC \\
57858.210 & 99.99 & $3 \times 30$ & Blue & 0.9 & LBT \\
57858.210 & 99.99 & $3 \times 30$ & Red & 0.9 & LBT \\
57873.903 & 115.68 & $2 \times 20$ & R1000B & 0.8 & GTC \\
57873.924 & 115.70 & $1 \times 20$ & R2500I & 0.8 & GTC \\
58142.431 & 384.21 & $4 \times 10$ & Blue & 1.2 & LBT \\
58142.431 & 384.21 & $4 \times 10$ & Red & 1.2 & LBT \\
58196.273 & 438.05 & $8 \times 10$ & Dual-grating & 0.7 & LBT \\
\hline
\end{tabular}

Notes. The columns are as follows: modified Julian date (1) and $\Delta t$ corresponding to the mid time of the observation (2), total exposure time (3), grism identification (4), average seeing of the night (5), and telescope used for the observation (6). ${ }^{(a)}$ This time is estimated from $T_{0}$.

similar to the observed evolution of the prototype supernova associated with long GRBs (SN 1998bw), peaking at similar time after the burst event ( 20 days). As for SN 1998bw, which was associated with a subluminous gamma-ray burst (GRB 980425), ATLAS17aeu was also found to be associated with a long under-energetic event (GRB 170105A). All our observations including the host galaxy ones point to the scenario of a long GRB at $z \sim 0.5$ unrelated to gravitational wave signal.

By assuming the fluence measured by Konus-Wind and, based on the soft spectrum inferred from the measurements by 
Table 3. Multi-band photometry of the host galaxy.

\begin{tabular}{ccc}
\hline \hline Filter & Magnitude (error) & Telescope \\
\hline F390W & $25.2 \pm 0.2$ & HST \\
$g$ & $24.69 \pm 0.11$ & LBT \\
F606W & $24.5 \pm 0.2$ & HST \\
$r$ & $24.38 \pm 0.08$ & LBT \\
$i$ & $24.14 \pm 0.06$ & LBT \\
$z$ & $24.02 \pm 0.04$ & GTC \\
F140W & $23.7 \pm 0.2$ & HST \\
\hline
\end{tabular}

Notes. The columns are as follows: filter identification (1), calibrated $\mathrm{AB}$ magnitude not corrected for Galactic extinction (2), and telescope used for the observation (3).

Konus-Wind, POLAR, and AstroSat-CZTI, a rest-frame spectral peak energy $E_{\mathrm{p}}$ of $50 \pm 25 \mathrm{keV}$, we find that GRB $170105 \mathrm{~A}$ would be consistent with the $E_{\mathrm{p}}-E_{\text {iso }}$ correlation of long GRBs (Amati et al. 2002; Amati 2006) only for $z>0.4-0.5$ (implying an isotropic energy for this event of $E_{\text {iso }} \gtrsim 2 \times 10^{51} \mathrm{erg}$ ). This finding further supports the above conclusion that this event came from a greater distance with respect to GW 170104, and is consistent with its association with a supernova at $z \sim 0.5$.

Acknowledgements. We thank the anonymous referee for the valuable comments and suggestions that improved the paper. We thank P. Shawhan for useful comments. AM acknowledges the support from the ASI grant I/004/11/3. AR acknowledges support from Premiale LBT 2013. AJCT thanks the Spanish Ministry Project AYA2015-71718-R (including FEDER funds). LT and SB are partially supported by the PRIN-INAF 2016 with the project "Toward the SKA and CTA era: discovery, localisation, and physics of transient sources". Partially based on observations collected at the Copernico $1.82 \mathrm{~m}$ telescope (Asiago, Italy) of the INAF - Osservatorio Astronomico di Padova. JH was supported by a VILLUM FONDEN Investigator grant (project number 16599). AFV is thankful to the Russian Science Foundation (grant 14-50-00043). This work made use of observations obtained with the Italian $3.6 \mathrm{~m}$ Telescopio Nazionale Galileo (TNG) and the $10.4 \mathrm{~m}$ Gran Telescopio Canarias (GTC), operated on the island of La Palma by the Fundación Galileo Galilei of the Instituto Nazionale di Astrofisica (INAF) at the Spanish Observatorio del Roque de los Muchachos of the Instituto de Astrofísica de Canarias, and also made use of observations obtained with the $8.4 \mathrm{~m}$ Large Binocular Telescope (LBT). The LBT is an international collaboration among institutions in Italy, the United States, and Germany. LBT Corporation partners are: Istituto Nazionale di Astrofisica, Italy; The University of Arizona on behalf of the Arizona university system; LBT Beteiligungsgesellschaft, Germany, representing the Max-Planck Society, the Astrophysical Institute Potsdam, and Heidelberg University; The Ohio State University; and The Research Corporation on behalf of The University of Notre Dame, University of Minnesota, and University of Virginia. We thank the TNG staff, in particular G. Andreuzzi, G. Mainella, A. Harutyunyan, and the LBT staff, in particular A. Gargiulo, for their valuable support with TNG and LBT observations and data reduction. We also acknowledge INAF financial support of the project "Gravitational Wave Astronomy with the first detections of adLIGO and adVIRGO experiments".

\section{References}

Abbott, B. P., Abbott, R., Abbott, T. D., et al. 2016a, Phys. Rev. Lett., 116, 061102

Abbott, B. P., Abbott, R., Abbott, T. D., et al. 2016b, Phys. Rev. Lett., 116, 241103

Abbott, B. P., Abbott, R., Abbott, T. D., et al. 2017a, Phys. Rev. Lett., 118, 221101

Abbott, B. P., Abbott, R., Abbott, T. D., et al. 2017b, Phys. Rev. Lett., 119, 141101

Abbott, B. P., Abbott, R., Abbott, T. D., et al. 2017c, ApJ, 851, L35

Abbott, B. P., Abbott, R., Abbott, T. D., et al. 2017d, Phys. Rev. Lett., 119, 161101

Abbott, B. P., Abbott, R., Abbott, T. D., et al. 2017e, ApJ, 848, L12
Amati, L. 2006, MNRAS, 372, 233

Amati, L., Frontera, F., Tavani, M., et al. 2002, A\&A, 390, 81

Arnouts, S., Cristiani, S., Moscardini, L., et al. 1999, MNRAS, 310, 540 Bhalerao, V., Kasliwal, M. M., Battacharya, D., et al. 2017a, ApJ, 845, 152 Bhalerao, V., Battacharya, D., Rao, A. R., \& Vadawale, S. 2017b, GRB Coordinates Network, 20412

Bloom, J. S., Kulkarni, S. R., Djorgovski, S. G., et al. 1999, Nature, 401, 453

Brocato, E., Castellani, V., Raimondo, G., et al. 1999, ApJ, 527, 230

Bufano, F., Pian, E., Sollerman, J., et al. 2012, ApJ, 753, 67

Calzetti, D., Armus, L., Bohlin, R. C., et al. 2000, ApJ, 533, 682

Campana, S., Mangano, V., Blustin, A. J., et al. 2006, Nature, 442, 1008

Cano, Z., Bersier, D., Guidorzi, C., et al. 2011a, ApJ, 740, 41

Cano, Z., Bersier, D., Guidorzi, C., et al. 2011b, MNRAS, 413, 669

Castro-Tirado, A. J., \& Gorosabel, J. 1999, A\&AS, 138, 449

Castro-Tirado, A. J., Sokolov, V. V., Gorosabel, J., et al. 2001, A\&A, 370, 398

Corsi, A., Kasliwal, M. M., Frail, D. A., \& Palliyaguru, N. T. 2017, GRB Circular Network, 20396, 1

D'Avanzo, P., Salvaterra, R., Sbarufatti, B., et al. 2012, MNRAS, 425, 506

Della Valle, M., Malesani, D., Benetti, S., et al. 2003, A\&A, 406, L33

Della Valle, M., Malesani, D., Bloom, J. S., et al. 2006, ApJ, 642, 103

D'Elia, V., Pian, E., Melandri, A., et al. 2015, A\&A, 577, A116

Evans, P. A., Kennea, J. A., Barthelmy, S. D., et al. 2017a, GRB Circular Network, 20390, 1

Evans, P. A., Beardmore, A. P., Page, K. L., et al. 2017b, GRB Circular Network, 20415, 1

Ferrero, P., Kann, D. A., Zeh, A., et al. 2006, A\&A, 457, 857

Feulner, G., Goranova, Y., Drory, N., et al. 2005, MNRAS, 358, 1

Galama, T. J., Vreeswijk, P. M., van Paradijs, J., et al. 1998, Nature, 395, 670

Galama, T. J., Tanvir, N., Vreeswijk, P. M., et al. 2000, ApJ, 536, 185

Greiner, J., Klose, S., Salvato, M., et al. 2003, ApJ, 599, 1223

Hjorth, J., Sollerman, J., Moller, P., et al. 2003, Nature, 423, 847

Horne, K. 1986, PASP, 98, 609

Hunt, L. K., Palazzi, E., Michałowski, M. J., et al. 2014, A\&A, 565, A112

Ilbert, O., Arnouts, S., McCracken, H. J., et al. 2006, A\&A, 457, 841

Japelj, J., Vergani, S. D., Salvaterra, R., et al. 2016, A\&A, 590, A129

Japelj, J., Vergani, S. D., Salvaterra, R., et al. 2018, A\&A, 617, A105

Jin, Z.-P., Covino, S., Della Valle, M., et al. 2013, ApJ, 774, 114

Kasliwal, M., Adams, S., Vedantham, H., et al. 2017, GRB Coordinates Network, 20393

Komatsu, E., Smith, K. M., Dunkley, J., et al. 2011, ApJS, 192, 18

Larson, D., Dunkley, J., Hinshaw, G., et al. 2011, ApJS, 192, 16

Malesani, D., Tagliaferri, G., Chincarini, G., et al. 2004, ApJ, 609, 5

Marcinkovski, R., Xiao, H., \& Hajdas, W. 2017, GRB Coordinates Network Circular Service, 20387

Melandri, A., Pian, E., Ferrero, P., et al. 2012, A\&A, 547, A82

Melandri, A., Pian, E., D'Elia, V., et al. 2014, A\&A, 567, A29

Mooley, K. P., Fender, R. P., Horesh, A., et al. 2017, GRB Circular Network, 20425, 1

Nava, L., Salvaterra, R., Ghirlanda, G., et al. 2012, MNRAS, 421, 1256

Patat, F., Cappellaro, E., Danziger, J., et al. 2001, ApJ, 555, 900

Pian, E., Mazzali, P. A., Masetti, N., et al. 2006, Nature, 442, 1011

Pogge, R. W., Atwood, B., Brewer, D. F., et al. 2010, Proc. SPIE, 7735, 77350A

Racusin, J. L., Liang, E. W., Burrows, D. N., et al. 2009, ApJ, 698, 43

Raimondo, G. 2009, ApJ, 700, 1247

Rossi, A., Piranomonte, S., Savaglio, S., et al. 2014, A\&A, 572, A47

Sari, R., Piran, T., \& Narayan, R. 1998, ApJ, 497, L17

Sari, R., Piran, T., \& Halpern, J. P. 1999, ApJ, 519, L17

Schlafly, E. F., \& Finkbeiner, D. P. 2011, ApJ, 737, 103

Schulze, S., Klose, S., Björnsson, G., et al. 2011, A\&A, 526, A23

Schulze, S., Malesani, D., Cucchiara, A., et al. 2014, A\&A, 566, A102

Sharma, V., Bhalerao, V., Battacharya, D., Rao, A. R., \& Vadawale, S. 2017, GRB Coordinates Network Circular Service, 20389

Soderberg, A. M., Kulkarni, S. R., Price, P. A., et al. 2006, ApJ, 636, 391

Sparre, M., Sollerman, J., Fynbo, J. P. U., et al. 2011, ApJ, 735, 24

Stalder, B., Tonry, J., Smartt, S. J., et al. 2017, ApJ, 850, 149

Svinkin, D., Golenetskii, S., Aptekar, R., et al. 2017, LIGO/Virgo Coordinates Network Circular Service, 20406

The LIGO Scientific Collaboration \& Virgo Collaboration 2017, Circular Service, 20364

Tonry, J., Denneau, L., Heinze, A., et al. 2017, LIGO/Virgo Coordinates Network Circular Service, 20382

Valenti, S., Benetti, S., Cappellaro, E., et al. 2008, MNRAS, 383, 1485

Vergani, S. D., Salvaterra, R., Japelj, J., et al. 2015, A\&A, 581, 102

Waters, C. Z., Zepf, S. E., Lauer, T. R., et al. 2006, ApJ, 650, 885

Willingale, R., Starling, R. L. C., Beardmore, A. P., Tanvir, N. R., \& O’Brien, P. T. 2013, MNRAS, 431, 394

Zeh, A., Klose, S., \& Hartmann, D. H. 2004, ApJ, 609, 952 